\title{
PALEO
}

Revue d'archéologie préhistorique

Comment ont été fendus les nodules ovoïdes de silex de la minière de ri (orne) ? Une reconstitution expérimentale de la fracture en split sur percuteur dormant à touche linéaire

How were the flint ovoid nodules from the Ri quarry (Orne, France) split? An experimental reconstruction of split fracture on resting hammer with linear striking surface

\section{Sébastien Giazzon}

\section{(2) OpenEdition}

\section{Journals}

Édition électronique

URL : http://journals.openedition.org/paleo/1974

DOI : $10.4000 /$ paleo. 1974

ISSN : 2101-0420

Éditeur

SAMRA

Édition imprimée

Pagination : 181-187

ISSN : 1145-3370

Référence électronique

Sébastien Giazzon, «Comment ont été fendus les nodules ovoïdes de silex de la minière de ri (orne) ? Une reconstitution expérimentale de la fracture en split sur percuteur dormant à touche linéaire » PALEO [En ligne], Numéro spécial | 2009-2010, mis en ligne le 24 avril 2012, consulté le 07 juillet 2020 URL : http://journals.openedition.org/paleo/1974 ; DOI : https://doi.org/10.4000/paleo.1974

\section{(c) (i) $\odot$}

PALEO est mis à disposition selon les termes de la licence Creative Commons Attribution - Pas d'Utilisation Commerciale - Pas de Modification 4.0 International. 


\title{
COMMENT ONT ÉTÉ FENDUS LES NODULES OVOÏDES DE SILEX DE LA MINIÈRE DE RI (ORNE) ? UNE RECONSTITUTION EXPÉRIMENTALE DE LA FRACTURE EN SPLIT SUR PERCUTEUR DORMANT À TOUCHE LINÉAIRE
}

\author{
Sébastien GIAZZON ${ }^{(1)}$
}

\begin{abstract}
Résumé : Une minière de silex néolithique à haches a été récemment fouillée sur près de deux hectares à Ri, dans le nord de l'Orne, par une équipe de l'Inrap dirigée par C. Marcigny. Les centaines de structures d'extraction fouillées, depuis de simples fosses jusqu'à des puits et galeries de six mètres de profondeur, ont exploité un banc de calcaire crayeux jurassique riche en nodules de silex arrondis et à cortex épais. Parallèlement à l'étude en cours du riche mobilier osseux et lithique, des programmes de reconstitution expérimentale ont été initiés, dont l'un porte sur les aspects qualitatifs et quantitatifs de la fabrication des têtes de haches.

L'entame de tels nodules s'est avérée poser un réel problème devant l'échec de la solution la plus évidente consistant à jeter un nodule comme percuteur contre un autre placé au sol. Il a été alors imaginé de les fendre en les projetant sur l'arête d'une enclume en silex, l'arête jouant comme un coin dans l'épaisseur du cortex. Cette technique s'est révélée efficace, moyennant une bonne stabilité de l'enclume et un surhaussement du tailleur pour assurer un impact suffisamment violent. L'incision de l'arête de l'enclume sur plusieurs centimètres provoque le fendage franc du nodule projeté selon un plan de fracturation équatorial bien régulier, comme cela a été observé sur le matériel archéologique. Le mécanisme de cette technique est celui de la fracture en split, avec la spécification d'une arête faisant coin dans un cortex épais. En cela, elle rappelle la percussion lancée verticale à « touche linéaire " observée dans le Moustérien ancien des Tares (Faivre et al. 2009), de même que la technique de taille de meules en silex meulière (Turq 2009).

Mots-clés : Basse-Normandie, Néolithique, minière de silex, haches, nodules de silex, fracture en split.

Abstract: How were the flint ovoid nodules from the Ri quarry (Orne, France) split? An experimental reconstruction of split fracture on resting hammer with linear striking surface. A Neolithic flint mine was recently excavated on two hectares at Ri, in the north Orne (Lower Normandy), by an Inrap team directed by C. Marcigny. The hundreds of structures of extraction excavated, since simple pits to wells and six depth meters galleries, exploited a bench of Jurassic chalky limestone rich in nodules of flint rounded and with thick cortex. Parallel to the study of the lithic assemblage, programs of experimental reconstitution were initiated, especially one studying the qualitative and quantitative aspects of the axes manufacture.

Starts such nodules proved to pose a real problem in front of the failure of the most obvious solution: the jet of a nodule like striker against another placed at the ground. It was then imagined to split them by projecting them on the edge of a flint anvil, the edge playing like a corner in the thickness of the cortex. This technique appeared effective, with the help of a good stability of the anvil and a heightening of the tailor to ensure a sufficiently violent impact. The incision of the edge of the anvil on several centimetres causes the frank splitting of the nodule projected according to an equatorial plan of fractu ring regular good, as that was observed on the archaeological material. The mechanism of this technique is that of the Split fracture, with the specification of an edge making corner in a thick cortex. In that, she recalls the vertical launched percussion to "linear key" observed in old Moustérien of Tares (Faivre et al. 2009), just as the technique of size of grinding flint grinding stones (Turq 2009).
\end{abstract}

Key-words: Basse-Normandie, Neolithic, flint mine, axes, flint nodules, split fracture.

\section{INTRODUCTION}

Le sous-sol jurassique de la Plaine de Caen, dans le sud du Calvados et le nord de l'Orne, a été l'objet d'importantes extractions minières de silex pendant le Néolithique. Des puits ont été depuis longtemps reconnus à Soignolles,
Potigny et Soumont-Saint-Quentin (Calvados), mais seule la minière de Bretteville-le-Rabet a été véritablement explorée par Jean Desloges dans les années 1980 (Desloges 1999). Celle de Ri, reconnue depuis quelques décennies par des ramassages de nombreuses ébauches et outils, a fait l'objet d'une importante fouille préventive 


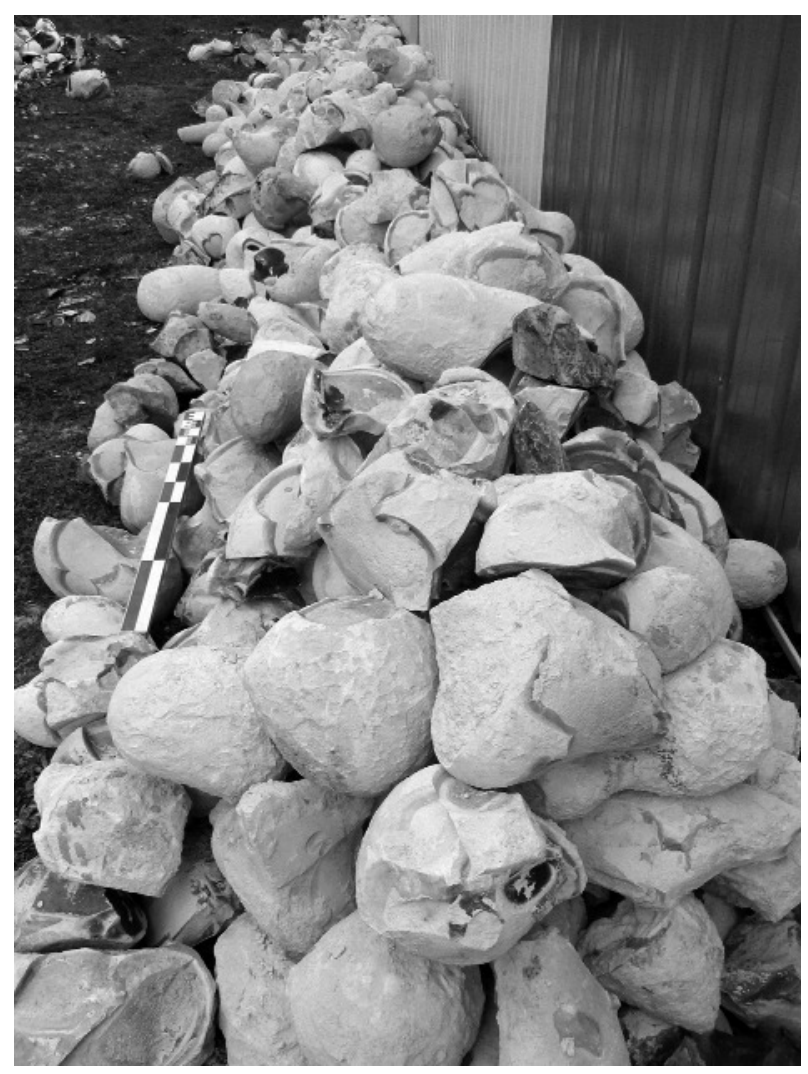

Figure 1 - Nodules de silex bruts, pour une part gélifractés, récupérés entre les puits fouillés de la minière de Ri (orne).

Figure 1 - Raw flint nodules, including frost weathered ones, recovered between the excavated pits of the flint quarry of Ri (Orne).

de juin à novembre 2007, liée à l'aménagement de l'autoroute A 88 (Marcigny et al. 2007).

La partie basse du site, en très faible pente, a été décapée sur $30 \mathrm{~m}$ de large et $600 \mathrm{~m}$ de longueur, révélant environ 600 puits d'extraction régulièrement espacés sur les près de deux hectares concernés. Ces structures ont exploité une nappe crayeuse d'une soixantaine de centimètres dans laquelle des nodules de silex sont disposés de manière anarchique. Vers le sud de l'emprise, là où le silex est sub-affleurant, il s'agit de fosses peu profondes avec de rares diverticules. Plus au nord, des puits de deux mètres de diamètre et de courtes galeries rayonnantes, fréquemment communicantes d'un puits à l'autre, ont été creusés jusqu'à deux mètres de profondeur. Vers l'extrémité nord, les puits les plus vastes prennent la forme d'un large entonnoir de quatre à 10 mètres de diamètre, tandis que d'autres descendent jusqu'à quatre à six mètres de profondeur et peuvent desservir deux à trois niveaux de galeries superposés. La plupart des puits ont livré des outils d'extraction en bois de cerf, de plusieurs types, et aussi quelques percuteurs du même matériau (matériel en cours d'étude, comme le mobilier lithique qui atteint sept tonnes).
Des lambeaux de sols piégés et des amas de restes de taille ont été rencontrés et fouillés, révélant que l'objectif principal de l'extraction était la fabrication de haches.

Parallèlement aux études en cours, différentes approches expérimentales ont été initiées. Un puits expérimental a été réalisé afin de comparer les marques observées dans les puits fouillés avec celles déterminées par différents outils d'extraction expérimentaux, ces derniers étant conservés comme référentiel tracéologique.

La reproduction expérimentale des opérations de taille, elle, a pour objectifs d'éclaircir les différentes options de taille retenues par les premiers exploitants et de participer à l'estimation quantitative du nombre de haches produites - ou productible - selon la conformation et la densité de la matière première.

C'est pourquoi, les nodules bruts récupérés entre les puits au cours de la fouille ont été systématiquement conservés dans l'optique de tests de taille après leur description (fig. 1). Dans la plus grande partie de la minière, ces nodules apparaissent disposés de façon anarchique au sein d'une matrice crayeuse formant une strate d'une soixantaine de centimètres.

\section{Des nodules bruts à tendance sphéroïde et à cortex épais}

Les nodules de silex de la minière de Ri présentent deux caractères assez originaux : leur morphologie et leur cortex. Ils affectent en effet une forme à tendance sphérique, faiblement étirée (ovoïde) ou pas, et rarement branchue. Les plus petits se présentent ainsi sous l'aspect d'un modeste boulet d'une dizaine de centimètres de diamètre, alors que les plus volumineux, en rognons arrondis, atteignent presque la quarantaine de centimètres.

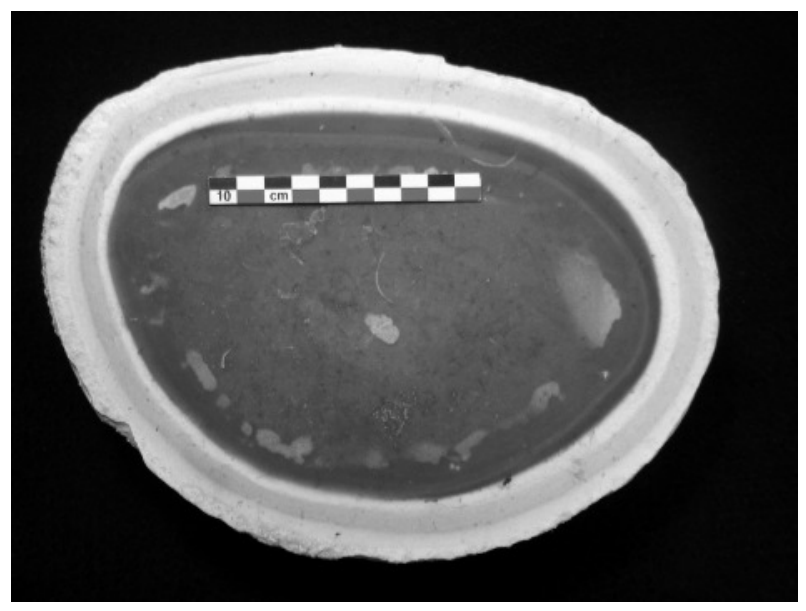

Figure 2 - Vue d'un module fendu montrant son cortex en trois couches : externe crayeuse, intermédiaire plus dure, interne à nouveau plus tendre.

Figure 2 - View of a split nodule showing its cortex with three layers : the outer chalky, the intermediate harder, and the inner softer one. 


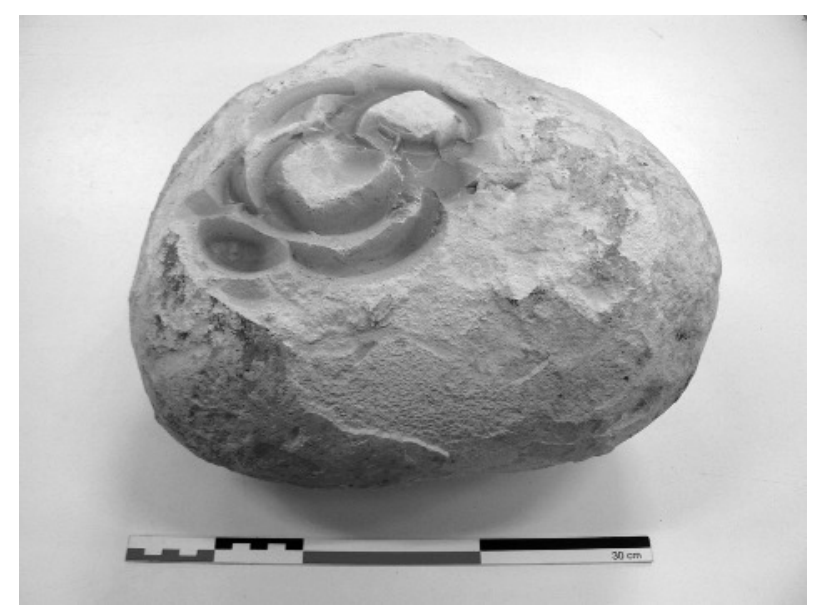

Figure 3 - Nodule brut plusieurs fois violemment percuté avec un autre nodule : sous le cortex desquamé apparaissent de larges cônes incipients.

Figure 3 - Raw nodule several times violently struck with another nodule : under the scaled cortex wide incipients cones appear.

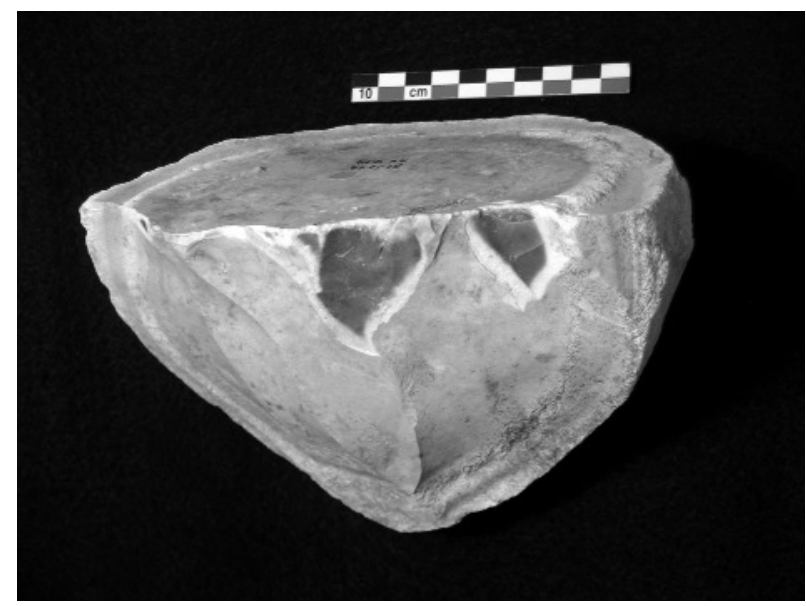

Figure 4 - Vue d'un des rares demi-nodules retrouvés fendus et abandonnés en début de dégrossissage, peutêtre un peu juste pour en tirer une préforme de hache.

Figure 4 - View of one of the rare half-nodules found split and abandonned at the beginning of roughing-out, perhaps because it was too small to make an axe preform.

Leur seconde particularité tient à leur enveloppe corticale très épaisse, de deux à trois centimètres. Elle se compose en fait de trois strates : une croûte externe d'aspect crayeux, une coque plus ou moins siliceuse plus dure et une couche à nouveau plus tendre en contact avec le silex exploitable (fig. 2). Ce cortex différencié est régulièrement présent sur l'ensemble des nodules. La gélifraction a parfois décalotté partiellement ou totalement certains rognons des deux premières couches de cette enveloppe.
On pressent ici que tant la forme sphéroïde sans angle de tels nodules que leur cortex épais vont compliquer leur entame, qui s'apparente alors à une " ouverture » critique sans laquelle il sera impossible de continuer...

Une fois la fouille achevée fin 2007 , les premiers essais expérimentaux de taille ont d'abord profité des quelques rognons déjà fendus ou fissurés par gélifraction. Le détachement de grands éclats de dégrossissage, pour façonner une ébauche tout en enlevant l'épais cortex, ne pose guère de difficultés sur ces rognons ouverts par le gel. Ces rognons consommés, les premiers essais d'ouverture de nodules entiers, en lançant un rognon sur un autre, se sont soldés par des échecs. Au lieu d'une entame franche, le rognon percuteur comme le percuté se marquent de profonds cônes incipients dont la répétition sur une même zone finissent par boucharder la surface du silex en y créant des fissures qui vont d'évidence compromettre la poursuite de la taille (fig. 3). Au mieux, après de nombreuses percussions qui ont d'abord décollé et écrasé le cortex, une esquille superficielle est détachée (esquille d'extérieur de cône incipient), dont le négatif convexe et ridé se prête mal au rôle de plan de frappe.

Après vérification, ni de telles esquilles d'extérieur de cône, ni de tels stigmates d'écrasements n'ont été observés dans les restes de taille archéologiques. À l'opposé, le talon bien lisse, large et épais de nombreux éclats archéologiques renvoyait à un plan de frappe initial régulier et plan, obtenu par une entame franche. C'est ce qu'indiquaient aussi les rares demi-nodules issus de la fouille : sans traces de cônes incipients et comme fragmentés selon un plan de fracture plan et régulier, sans concavité ou convexité bulbaire (fig. 4). II s'agissait donc bien d'une fracture en split, comme celle qui peut être obtenue par percussion verticale sur enclume, posée ou lancée, afin de fendre en deux des petits galets. Mais, pour ces gros nodules à cortex épais, il fallait trouver autre chose que le simple entrechoquement de 2 nodules l'un contre l'autre... Par ailleurs, la fouille n'a pas livré de percuteurs en roches tenaces.

C'est alors que nous avons pensé à fendre le nodule lancé non pas contre un autre nodule, mais sur une arête obtuse, dégagée d'un bloc de silex. Après mise au point assez rapide, nous allons voir que cette idée s'est révélée efficace et conforme au matériel archéologique.

\section{Reconstitution de la technique de fendage des rognons sphéroïdes de $\mathrm{Ri}$}

Plusieurs conditions sont apparues déterminantes pour la bonne efficacité de cette technique. La première tient à la confection d'un percuteur dormant particulier, en ce qu'il doit présenter une arête rectiligne longue d'au moins $6 \mathrm{~cm}$, selon un angle dièdre de $120^{\circ}$ à $130^{\circ}$. Une telle arête, sous forme d'une nervure entre deux négatifs, est obtenue en détachant un ou quelques gros éclats d'un gros nodule gélifracté, au percuteur lourd en percussion lancée (fig. 5 et 6). Sa mise en forme n'est pas toujours réussie lors des premières tentatives. Le percuteur dormant n'est pas nécessairement de grande dimension. Si sa masse propre 


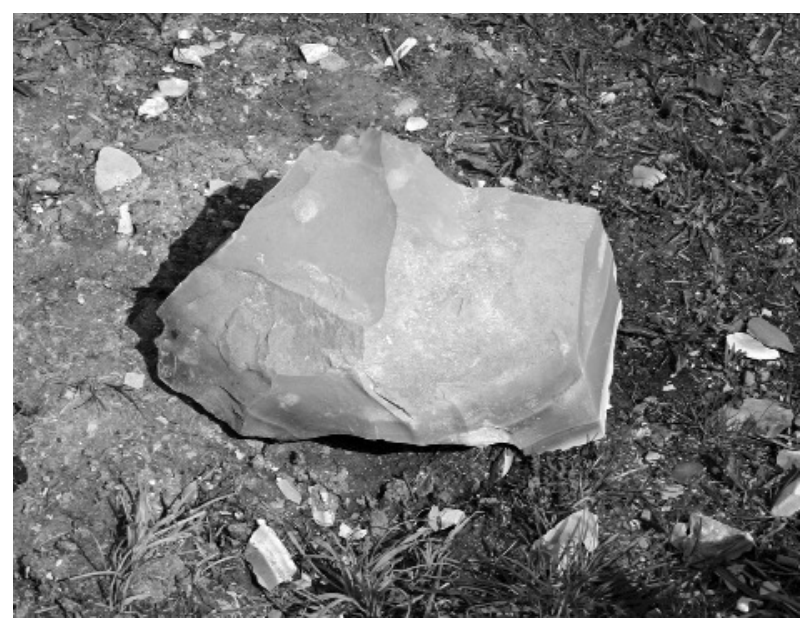

Figure 5 - Percuteur dormant expérimental « vierge » à arête obtuse, obtenue par le négatif d'un seul enlèvement sur un gros rognon gélifracté.

Figure 5 - Unused experimental resting hammer with obtuse edge, obtained by the negative of a single removal on a large frost weathered nodule.

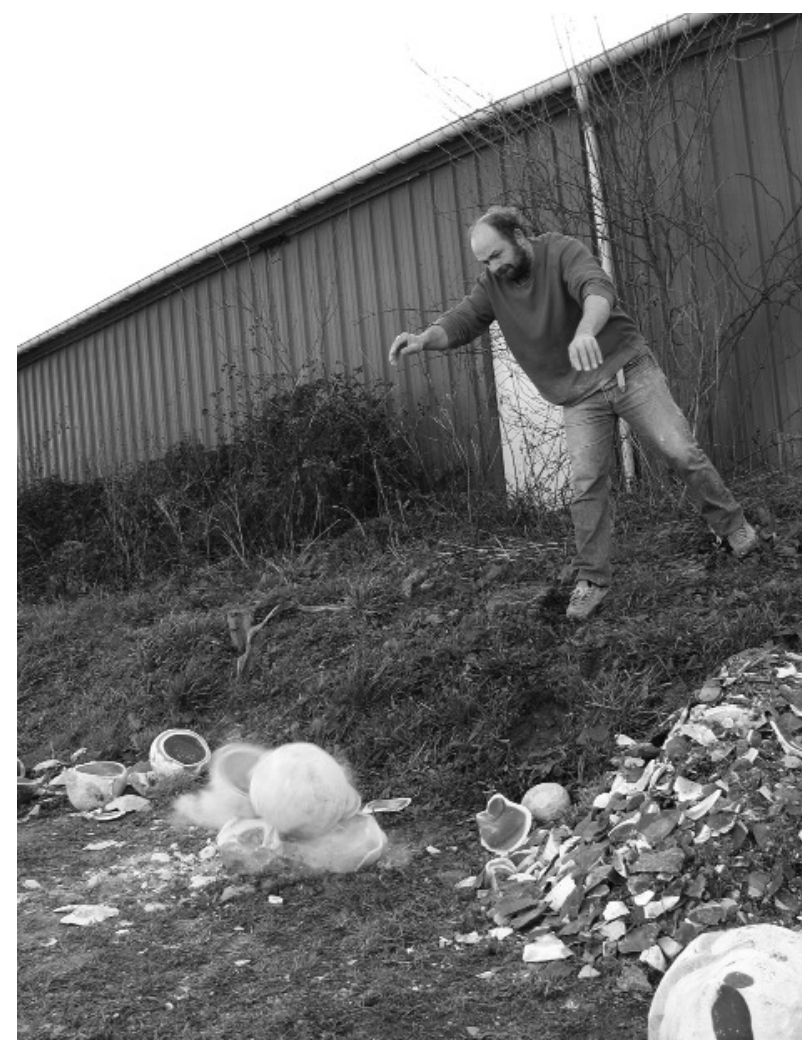

Figure 7 - Jet, par l'auteur, d'un nodule sphéroïde depuis le haut d'un talus sur un percuteur dormant à arête : fendage réussi en deux demi-nodules hémisphériques.

Figure 7 - Throwing by the author, of a spheroid nodule from the top of a slope on a resting hammer with edge : successful splitting in two hemispherical half-nodules.

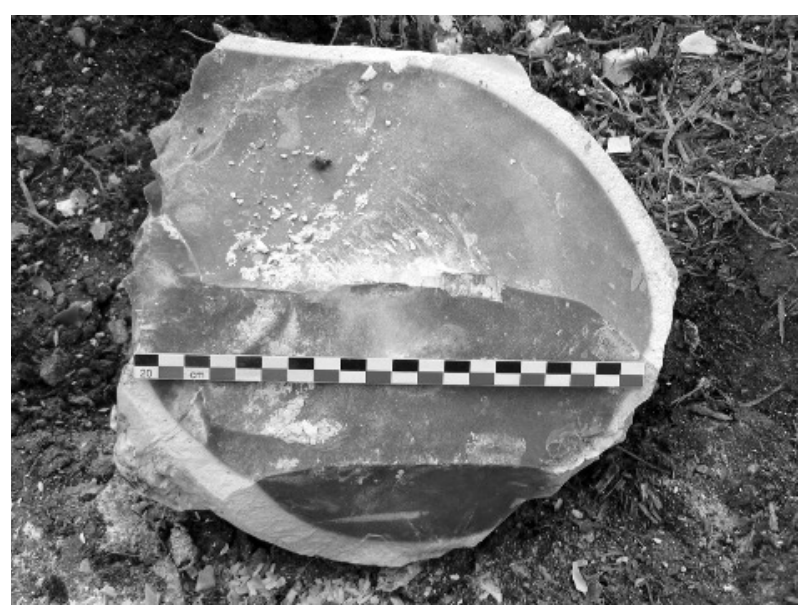

Figure 6 - Percuteur dormant expérimental à arête centrale créée par deux grands enlèvements, en cours d'usage.

Figure 6 - Experimental resting hammer with central edge created by two large removals, while being used.

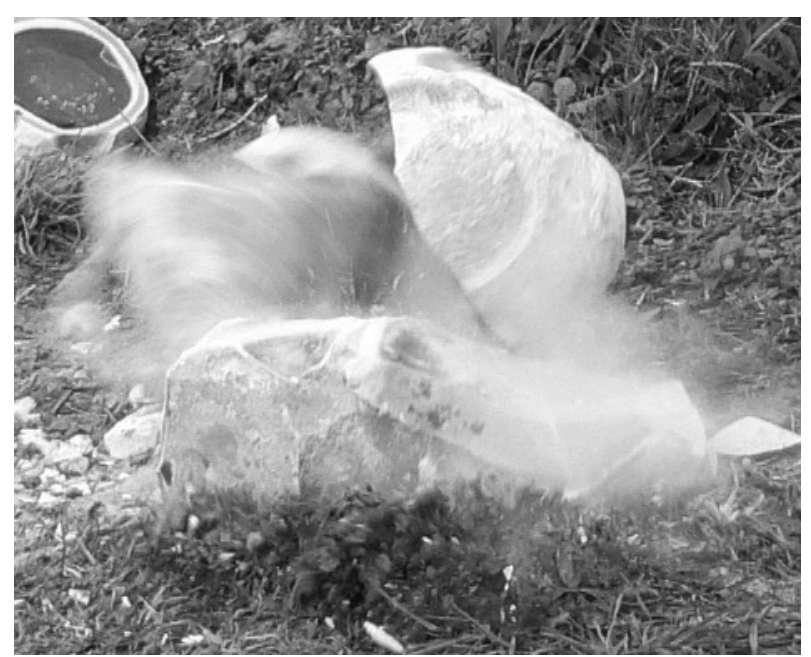

Figure 8 - Fendage net d'un nodule ovoïde, une fraction de seconde après l'impact : les deux fragments roulent jusqu'à deux mètres du percuteur dormant.

Figure 8 - Clear splitting of an ovoide nodule, a fraction of second after the impact : the two fragments roll up to two meters of the resting hammer. 


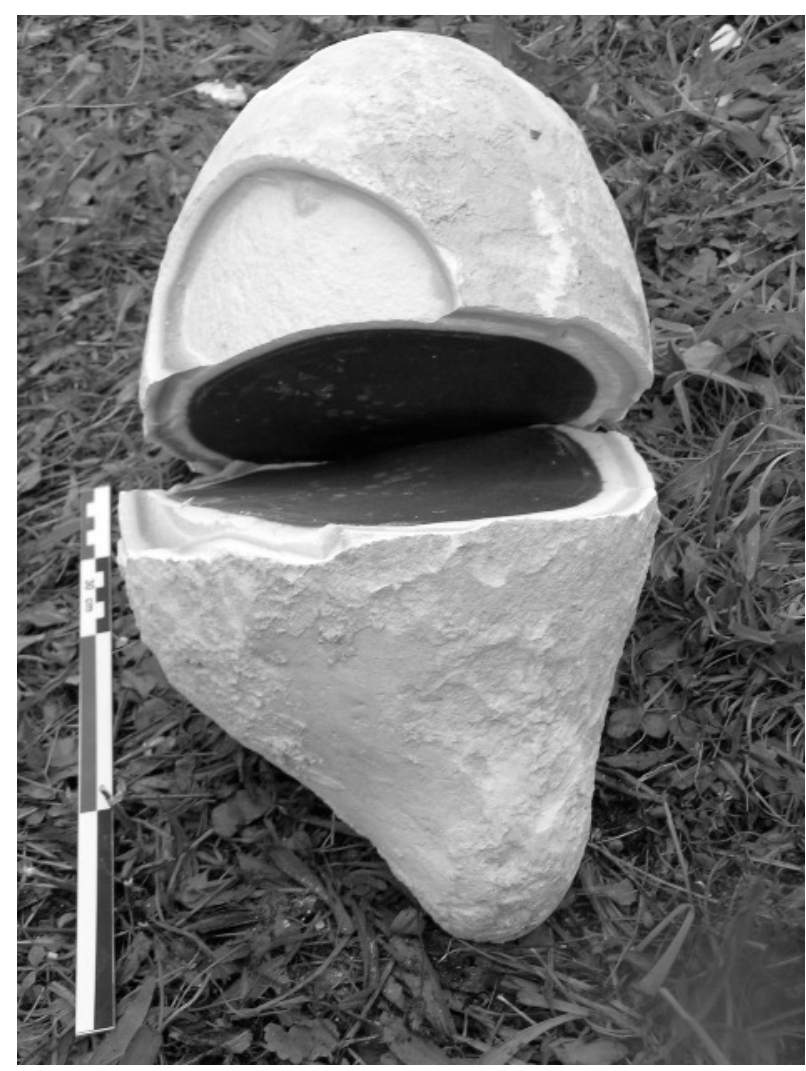

Figure 9 - Les deux fragments rapprochés du nodule ovoïde fendu en deux (fig. 8) : l'impact a porté au niveau de la tête de la règle, déterminant un plan de fracturation bien régulier.

Figure 9 - Two fragments of the split ovoid nodule (fig. 8) : the impact was applied at the level of the jalon head, determining a quite regular plan of fracturing.

n'est pas un facteur déterminant, sa stabilité au sol doit être rigoureuse pour un bon fendage des nodules bruts. Cette deuxième condition, la stabilité du percuteur dormant au sol, peut être assurée de deux façons : en sélectionnant pour sa face inférieure plate ou concave, ou encore en calant dans un aménagement sommaire sous forme d'une cuvette creusée au sol. La stabilité du percuteur dormant sous le choc assure une franche incision en coin de l'arête dans le cortex du nodule projeté et donc son effet de fendage. De plus, le soin apporté à cette mise en place évite une éventuelle dégradation de l'arête lors d'un lancer mal ciblé.

D'autres conditions d'optimisation concernent les nodules candidats au fendage. Ils doivent d'abord présenter une large surface corticale intacte, qui subira l'impact. En effet, l'épaisseur du cortex brut paraît capitale pour que la technique puisse se réaliser. Les nodules les plus aisés à fendre peuvent peser de quinze à plus de trente de kilos. Les plus réguliers sont les plus adaptés, autorisant une prise en main favorable à un lancer plus adroit. II apparait également que la symétrie relative du rognon facilite son fendage en deux moitiés relativement égales.
Une dernière condition tient à l'énergie cinétique nécessaire pour un bon fendage. L'énergie délivrée en guidant simplement la chute du nodule à fendre sur un percuteur dormant disposé devant soit s'est révélée insuffisante, l'impact ne produisant pas le fendage attendu. Pour augmenter cette énergie, il fallait atteindre une vitesse à l'impact plus élevée $\left(E=1 / 2 m v^{2}\right)$ et donc lancer le nodule de plus haut pour profiter de l'accélération déterminée par l'attraction terrestre (selon $\mathrm{g}$, une seconde de temps de chute augmente la vitesse de $9,1 \mathrm{~m} / \mathrm{s}$ ). Nous nous sommes donc rehaussé sur le haut d'un talus, nos pieds dominant le percuteur dormant d'un bon mètre, hauteur qui est apparue effectivement suffisante pour optimiser le taux de succès (fig. 7).

Il était aisé, pour les néolithiques de Ri, de se positionner de même sur un tas de déblais à proximité d'un puits en activité, ou même d'installer une sorte de plateforme surplombant une enclume scellée au fond d'une fosse pour y lâcher le nodule avec précision.

Le nodule lancé de plus haut doit encore atteindre l'arête de l'enclume avec précision, de façon à ce que l'impact sur l'arête porte à peu près sur l'équateur du nodule pour un fendage en deux moitiés ou tout au moins en deux fragments chacun destinés à être façonnés en une tête de hache. II y faut une certaine expérience, et un peu d'échauffement au début d'une nouvelle session. L'arête incise alors le cortex, et, si l'énergie est suffisante, y créée une fissuration linéaire qui se propage dans le silex, selon un plan vertical régulier (fig. 8 et 9 ). Ainsi, ce contact linéaire à la fois facilite le fendage et guide l'orientation de la fracture selon un plan équatorial, les deux pôles du nodule étant empaumés par les deux mains. Consécutivement à l'impact, les deux fragments roulent dans un rayon de un à trois mètres aux abords du percuteur dormant.

\section{Évaluation}

Cette technique de fendage détermine sur chaque fragment une surface de fracture tout à fait plate et bien régulière, idéale comme plan de frappe initial du façonnage ou débitage à suivre. Sur ce silex de grain moyen, les rides de propagation sont à peine discernables à l'œil, et, comme il s'agit d'une fracturation en split " pure ", il n'y a aucune concavité ou convexité en regard de la ligne d'impact, relief qui évoquerait un mécanisme ou une composante de fracture conchoïdale (fig. 9). Ce résultat est bien celui observé sur le matériel archéologique (fig. 4).

L'exercice de cette technique, sur une trentaine de nodules lors d'une première série de tests, n'a rencontré que très peu d'échecs, même si certains de ces rognons ne se sont pas fendus au premier impact et ont dû être relancés jusqu'à 4 ou 5 fois. Cela dit, nous sommes loin d'avoir toujours obtenu un fendage en deux moitiés à peu près égales, à cause de l'approximation du lancer qui peut faire tomber l'impact à proximité d'un pôle du nodule alors simplement décalotté d'un quart ou même moins (fig. 10). Quelques rares rognons ont refusé l'ouverture, perdant uniquement de larges esquilles corticales (fig. 11). Ces échecs paraissent dépendre du percuteur dormant instable et/ou d'un lancer peu adroit. 


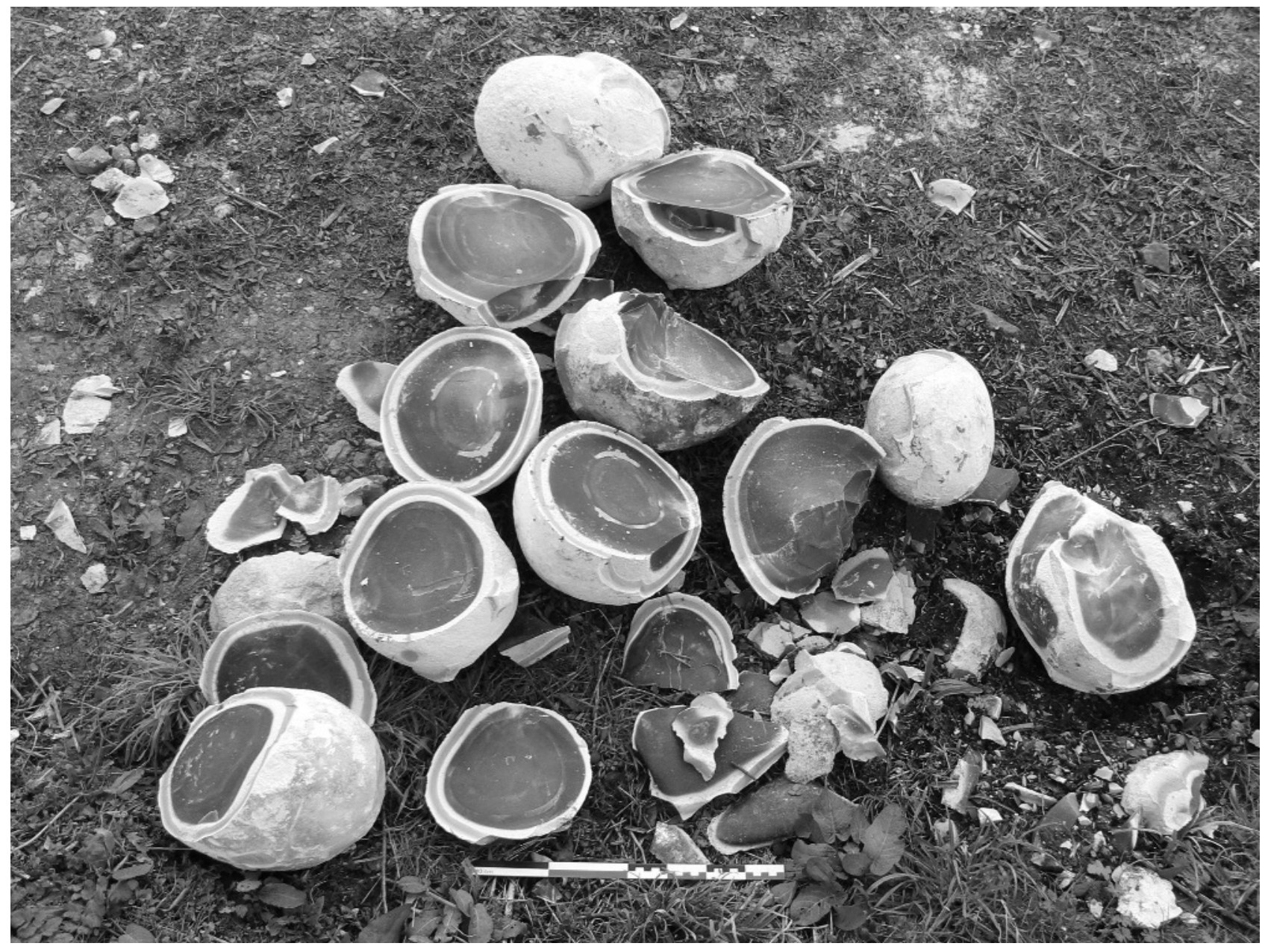

Figure 10 - Résultat d'une série de lancers : quatre nodules sont correctement fendus, plus ou moins symétriquement, deux nodules sont entamés mais quelque peu dégradés (effet d'un cône incipient préexistant ou d'une fissure de gel), les deux derniers ne se sont pas fendus mais l'un d'entre eux garde assez de cortex pour être relancé.

Figure 10 - Result of series of throwing : four nodules are correctly split, more or less symmetrically, two nodules are broken but somehow damaged (effect of a pre-existing cone or of a frost crack), the two last ones were not split but one of them keeps enough cortex to be thrown again).

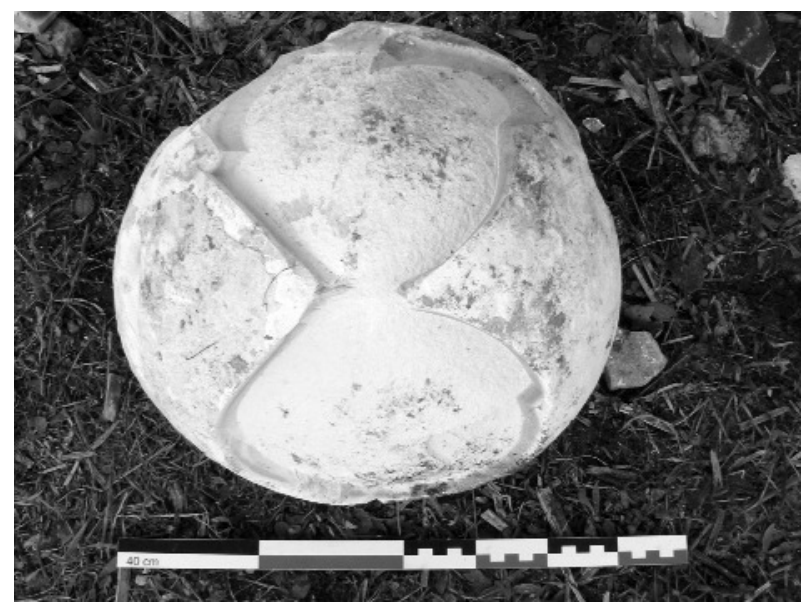

Figure 11 - Nodule lancé sur l'arête du percuteur dormant mais non fendu : l'impact a fait sauter une large esquille corticale de part et d'autre du contact linéaire.

Figure 11 - Nodule thrown on the edge of the resting hammer but not split: the impact detached a large cortical scar on both sides of the linear contact. 
L'arête du percuteur dormant, par son angulation obtuse, résiste assez bien à ce traitement, même si la formation d'un esquillement modéré est constaté (fig. 12). Cette dégradation ne semble pas affecter son efficacité lors des fendages ultérieurs. La durée de vie d'un percuteur dormant est difficile à déterminer, essentiellement dépendante de l'adresse de l'utilisateur. Les nôtres ont chacun permis le traitement d'une douzaine de rognons.

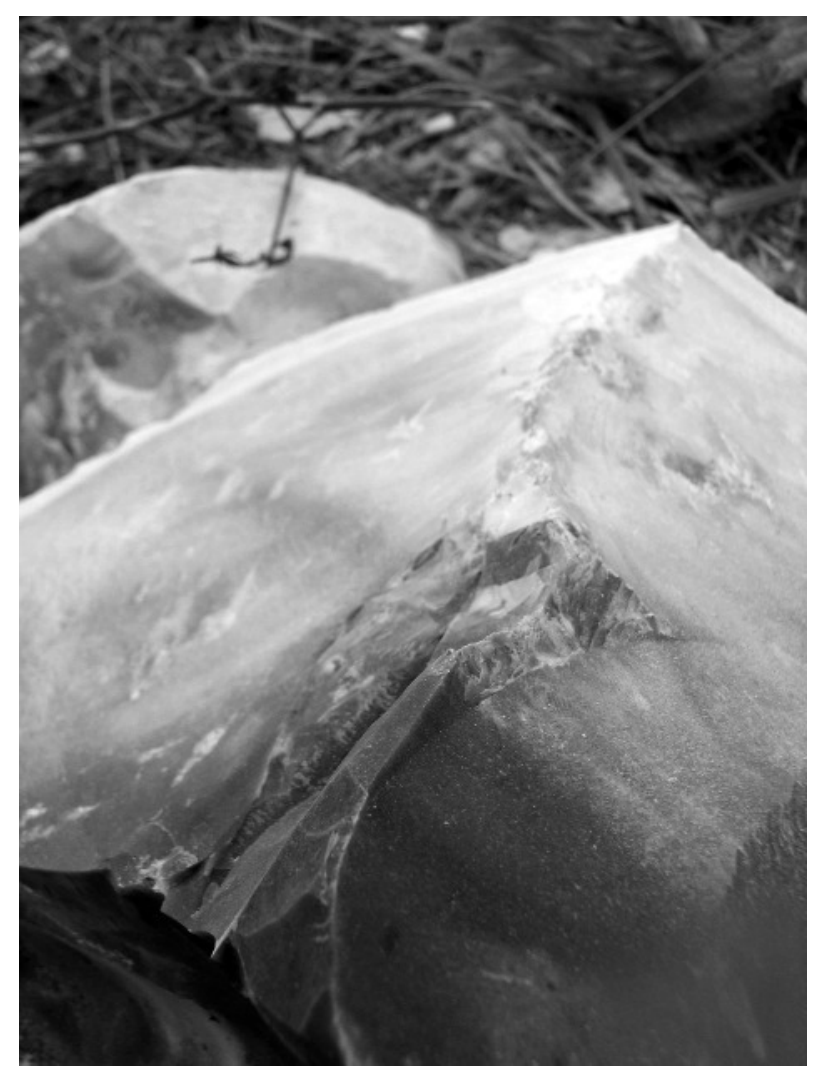

Figure 12 - Vue de l'arête, modérément esquillée, d'un percuteur dormant après fendage de plusieurs nodules.

Figure 12 - View of the moderately splintered edge, of a resting hammer, after splitting of several nodules.
Ainsi, au vu de son efficacité et de la similarité des stigmates obtenus avec le matériel archéologique, nous pensons que cette technique expérimentale est très proche sinon identique dans son principe à celle que les tailleurs néolithiques de la minière de Ri ont mise en jeu pour entamer les nodules sphéroïdes qui s'y trouvaient.

Sur le plan mécanique, cette technique met en jeu le mécanisme de la fracture en split (par fente verticale lancée ou posée sur enclume) mais avec la spécification d'une « touche linéaire » de l'impact, qui en améliore l'efficacité et le contrôle.

Elle s'apparente ainsi à la " percussion verticale à touche linéaire " déjà mise en jeu dans le gisement moustérien ancien des Tares (Faivre et al. 2009), et, bien plus tard et avec des outils de métal, à celle décrite par A. Turq (2009) pour la fragmentation et le façonnage des blocs de silexmeulière du plateau de Born près de Domme.

Je remercie Cyril Marcigny (Inrap et UMR 6566-CReAAH) et Jacques Pelegrin (UMR 7055 « Préhistoire et Technologie »), qui m'ont aidé dans la préparation de cet article.

\section{BIBLIOGRAPHIE}

J. DESLOGES 1999 - Une mine de silex au Néolithique, l'exemple de Bretteville-le-Rabet, in. San Juan G. et Maneuvrier J. (dir.), L'exploitation ancienne des roches dans le Calvados : histoire et archéologie, Couleurs Calvados, Molay-Littry, p. 53-78.

C. MARCIGNY, E. GHESQUIÈRE ET J. DESLOGES 2007 La Hache et la Meule, les premiers paysans du Néolithique en Normandie (6000-2000 avant notre ère), éd. Du Muséum du Havre, Le Havre, 191 p.

Photos de l'auteur, sauf figures 7 et 8 : photos L. Juhel. 
